\title{
Qualidade de vida: impactos de um programa de promoção da saúde do setor de saúde suplementar
}

\author{
Quality of life: impacts of a health promotion program \\ in the supplementary health sector
}

Hildegard Hedwig Pohl (https://orcid.org/0000-0002-7545-4862) ${ }^{1}$

Carmem Elisa Beschorner (https://orcid.org/0000-0002-0259-9059) ${ }^{1}$

Analie Nunes Couto (https://orcid.org/0000-0003-4819-5516) ${ }^{1}$

Tiago Henrique Lenhard (https://orcid.org/0000-0003-3371-6411) ${ }^{2}$

Polliana Radtke dos Santos (https://orcid.org/0000-0002-1721-4334) ${ }^{3}$

${ }^{1}$ Programa de PósGraduação em Promoção da Saúde, Universidade de Santa Cruz do Sul. Av. Independência 2.293, Bairro Universitário. 96815-900. Santa Cruz do Sul RS Brasil. hildegardpohl@ outlook.com

${ }^{2}$ Programa de PósGraduação em Economia, Universidade do Vale do Rio dos Sinos. São Leopoldo RS Brasil.

${ }^{3}$ Programa de Residência Multiprofissional em Saúde

- Urgência, Emergência e Intensivismo, Hospital Santa Cruz. Santa Cruz do Sul RS Brasil.

\begin{abstract}
The objective of this study was to compare quality of life indicators and clinical parameters among individuals participating in a cardiovascular health promotion program in the supplementary health sector. We conducted a cross-sectional study with 251 program participants living in the regions covered by the Vale do Taquari and Vale do Rio Pardo regional development councils in Rio Grande do Sul, Brazil. Quality of life was assessed using the WHOQOL-BREF questionnaire. Participants were predominantly women and elderly and had high cardiovascular risk (37.8\%) and good self-reported quality of life. The clinical parameter means were body mass index obesity class I, normal blood pressure and lipid profile and adequate fasting blood sugar level. The results of the reassessment after one year showed a significant reduction in mean triglycerides $(p=0.031)$, diastolic blood pressure $(p=0.000)$ and systolic blood pressure $(p=0.013)$, and a significant increase in the mean score for the general domain of the WHOQOL-BREF $(p=0.004)$. It is necessary to consider and address social determinants of health and promote integrated actions across various sectors, including both the public and private spheres.
\end{abstract}

Key words Health promotion, Quality of life, Chronic diseases, Supplementary health
Resumo O objetivo deste trabalho foi comparar indicadores da qualidade de vida e parâmetros clínicos de usuários que participam de um programa de promoção da saúde, no âmbito da saúde suplementar, na linha de cuidado cardiovascular. Trata-se de um estudo observacional e transversal, realizado com 251 sujeitos das regiões do Conselho Regional de Desenvolvimento Vale do Taquari e do Conselho Regional de Desenvolvimento Vale do Rio Pardo/RS. Para a avaliação da qualidade de vida, utilizou-se o questionário WHOQOL -BREF. Os participantes se caracterizaram pela predominância de mulheres, idosos, do aumento do número de sujeitos de alto risco cardiovascular $(37,8 \%)$ e de boa qualidade de vida. As médias dos parâmetros clínicos apontaram índice de massa corporal de obesidade grau I, pressão arterial normal, perfil lipídico e glicemia de jejum adequados. Após participarem durante um ano do programa, foram identificadas reduções significativas para as médias dos triglicerídeos ( $p=0,031)$, pressão arterial diastólica $(p=0,000)$, pressão arterial sistólica $(p=0,013)$, mas no domínio geral da qualidade de vida $(p=0,004)$ houve um aumento significativo. Se faz necessário considerar e intervir nos condicionantes sociais de saúde $e$ promover a intersetorialidade, inclusive entre $o$ público e o privado.

Palavras-chave Promoção da saúde, Qualidade de vida, Doenças crônicas, Saúde suplementar 


\section{Introdução}

Nas últimas décadas, o fenômeno do envelhecimento populacional vem avançando rapidamente no mundo, principalmente em países desenvolvidos ou em desenvolvimento, como é o caso do Brasil. Segundo o último Censo (2010), o Brasil conta com uma população de 20.588 .890 pessoas com mais de 60 anos de idade. Essa população, geralmente apresenta múltiplas doenças crônicas, que perduram por anos, exigindo assistência constante, medicação contínua e exames frequentes. Entre os agravos, destacam-se a hipertensão arterial (53\%), artrites (24\%), doenças cardiológicas (17\%), diabetes (16\%) e depressão (12\%), com $69 \%$ da população idosa apresentando pelo menos uma destas doenças ${ }^{1-3}$.

As demandas provenientes dessas patologias oneram e consomem parcela significativa do orçamento da saúde, impactando na sustentabilidade dos sistemas de saúde, público ou suplementar, além de contribuírem negativamente na produtividade e na qualidade de vida $(\mathrm{QV})$ das populações. Nesse sentido, as políticas públicas direcionam investimentos como medidas de prevenção de risco e de doenças, implementando ações de promoção da saúde em todos os ciclos da vida. Cabe considerar que essas devem ser adequadas às múltiplas realidades sociais, em consonância com as diferenças demográficas, epidemiológicas, socioeconômicas e culturais que caracterizam o país ${ }^{4,5}$.

Em 2011, o Brasil lançou o Plano de Ações Estratégicas para o Enfrentamento das Doenças Crônicas não Transmissíveis, 2011-2022. Nesse plano foram abordados quatro principais grupos de doenças (cardiovasculares, câncer, respiratórias crônicas e diabetes) e seus fatores de risco modificáveis comuns nesses agravos (tabagismo, álcool, inatividade física, alimentação inadequada e obesidade), bem como definidas três diretrizes estratégicas de atuação: (a) vigilância, informação, avaliação e monitoramento, (b) promoção da saúde, (c) cuidado integral de doenças crônicas não transmissíveis ${ }^{6-9}$.

Promover saúde é um processo extremamente complexo e exige dos profissionais da saúde o desenvolvimento de novas estratégias de atenção por meio da inovação, tendo claro a dimensão cuidadora da saúde ${ }^{10,11}$. Assim, mensurar a QV se torna um importante indicador para o planejamento de ações em saúde, pois pode indicar a percepção do indivíduo de sua posição na vida no contexto da cultura e do sistema de valores nos quais ele vive, assim como em relação a seus objetivos, expectativas, padrões e preocupações, refletindo e respeitando a diversidade de cada região e visando a diminuição das assimetrias em saúde ${ }^{12-14}$.

A utilização da QV como indicador de saúde ainda é pouco difundida, restringindo-se a grupos específicos, em geral no âmbito da saúde pública. Quando analisamos o setor suplementar de saúde, menos informações de saúde são disponibilizadas, salvo as oferecidas pela Agência Nacional de Saúde Suplementar (ANS), relacionadas a origens alicerçadas em um modelo com foco no viés econômico e financeiro, não obstante $27 \%$ da população brasileira ser usuária desse setor ${ }^{15}$.

Gradualmente, essa realidade está mudando, tanto pelo aumento das demandas dos usuários como pela atuação da ANS desde o ano 2000. Por meio de legislação específica, vem regulando o setor e buscando um alinhamento entre as políticas públicas e as do setor suplementar. A partir de 2004, a ANS vem incentivando o desenvolvimento de programas de promoção da saúde, que, associado às novas demandas, promoveu a ampliação de seus investimentos em promoção da saúde e no cuidado integral à saúde de seus clientes ${ }^{5,16}$.

$\mathrm{Na} 1^{a}$ Conferência Internacional de Promoção da Saúde, em 1986, com a apresentação da Carta de Ottawa, foi introduzido o conceito de promoção da saúde como "processo de capacitação da comunidade para atuar na melhoria da sua qualidade de vida e saúde..... A QV auxilia na constatação de morbidades ou riscos, além de dificuldades para a saúde mental ${ }^{17}$. Investir em QV requer conhecer a percepção dos usuários, para que haja contribuições efetivas.

Diante desse cenário, o objetivo do presente estudo foi comparar indicadores da QV e parâmetros clínicos de usuários que participam de um programa de promoção da saúde, no âmbito da saúde suplementar, na linha de cuidado cardiovascular.

\section{Método}

Trata-se de um estudo observacional e transversal que utilizou dados dos prontuários dos usuários do sistema suplementar de saúde em dois momentos. Esses usuários integravam um programa de promoção da saúde no âmbito do cuidado cardiovascular. $\mathrm{O}$ ingresso dos usuários ocorreu por demanda espontânea, por prescrição médica ou pela busca ativa ao serviço. Tal ingresso foi precedido pelo instrumento de avaliação da qualidade de vida abreviado (WHOQOL-BREF) 
e acompanhado por um plano terapêutico individual, composto por diferentes intervenções, como: consultas multiprofissionais, oficinas, palestras, grupos, encontros, atividades físicas e monitoramentos telefônicos.

Foram critérios de inclusão na seleção dos prontuários: tratar-se de usuários que responderam ao instrumento de avaliação da qualidade de vida abreviado (WHOQOL-BREF) em dois momentos, primeiro ao ingressar no programa, depois um ano após o ingresso. Além de terem realizado a avaliação global de risco cardiovascular proposta pelo programa. Atenderam aos critérios de inclusão todos os adultos e idosos, totalizando 251 sujeitos. Não foram considerados para o estudo os indivíduos menores de 18 anos, pois não possuíam a avaliação global do risco cardiovascular e respostas do questionário WHOQOL-BREF.

O projeto de promoção de saúde da instituição de saúde complementar foi implantado em quatro municípios: Lajeado e Encantado, que integram a região do Conselho Regional de Desenvolvimento do Vale do Taquari/RS (COREDE/ VT), neste estudo identificada como Região 1; além dos municípios de Santa Cruz do Sul e Venâncio Aires, pertencentes ao COREDE do Vale do Rio Pardo/RS (COREDE/VR), denominados nesta pesquisa como Região 2.

Os municípios de Encantado e Lajeado, da Região 1, classificada em segundo lugar no ranking do Índice de Desenvolvimento Socioeconômico (IDESE) do Rio Grande do Sul, destacam-se nos itens educação e saúde, respectivamente. Já os municípios de Venâncio Aires e Santa Cruz do Sul, da Região 2, caracterizada pela presença da produção e da indústria de tabaco, apresentam renda per capita maior que a Região 1 . Todos os municípios apresentam IDESE classificado como alto, exceto Venâncio Aires, classificado como médio $^{18}$.

Para a elaboração do perfil dos usuários, foram selecionadas nos prontuários as variáveis: idade, sexo, índice de massa corporal $\left(\mathrm{IMC}=\mathrm{Kg} / \mathrm{m}^{2}\right.$ ), perfil lipídico, glicemia de jejum (GLI), pressão arterial sistólica (PAS) e diastólica (PAD), classificação do risco cardiovascular e o questionário WHOQOL-BREF.

O IMC foi classificado segundo a Organização Mundial da Saúde ${ }^{9}$, que descreve o IMC como baixo peso (IMC $<18,5 \mathrm{Kg} / \mathrm{m}^{2}$ ), eutrófico (IMC $\left.18,5-24,9 \mathrm{~kg} / \mathrm{m}^{2}\right)$, sobrepeso $\left(25-29,9 \mathrm{~kg} / \mathrm{m}^{2}\right)$, obesidade grau I $\left(30,0\right.$ a $\left.34,9 \mathrm{Kg} / \mathrm{m}^{2}\right)$, obesidade grau II $\left(35,0\right.$ a $\left.39,9 \mathrm{Kg} / \mathrm{m}^{2}\right)$ e obesidade grau III $(\geq 40,0$ $\left.\mathrm{Kg} / \mathrm{m}^{2}\right)$.
Para a determinação do perfil lipídico foram analisadas as variáveis: colesterol total (CT), lipoproteína de baixa densidade (LDL-c), lipoproteína de alta densidade (HDL-c) e triglicerídeos (TRI). Para a classificação dessas variáveis, assim como para definir o risco cardiovascular, foram considerados os pontos de corte proposto na $5^{\mathrm{a}}$ Diretriz Brasileira Sobre Dislipidemias e Prevenção da Aterosclerose da Sociedade Brasileira de Cardiologia $(\mathrm{SBC})^{19}$. Esta classifica como baixo risco de apresentar os principais eventos cardiovasculares em dez anos (doença arterial coronariana - DAC, acidente vascular cerebral - AVC, doença arterial obstrutiva periférica ou insuficiência cardíaca) aqueles com probabilidade $<5 \%$. No entanto, na presença de histórico familiar de doenças cardiovasculares precoces, os classificados com baixo risco foram reclassificados como com risco intermediário. Foram classificados com risco intermediário homens com risco calculado $\geq 5 \%$ e $\leq 20 \%$ e mulheres com risco calculado $\geq 5 \%$ e $\leq 10 \%$. Foram considerados de alto risco homens com risco calculado $>20 \%$ e mulheres $>10 \%$ no período de dez anos.

Em relação ao CT, foi considerado alto quando $\geq$ que $240 \mathrm{mg} / \mathrm{dl}$, limítrofe quando 200 $239 \mathrm{mg} / \mathrm{dl}$, e ótimo quando $<200 \mathrm{mg} / \mathrm{dl}$. O HDL-c foi considerado baixo quando $<40 \mathrm{mg} / \mathrm{dl}$ e desejável quando $>60 \mathrm{mg} / \mathrm{dl}$. O LDL-c $<100 \mathrm{mg} / \mathrm{dl}$ foi classificado como ótimo, de 100-129 mg/dl foi desejável, de 130-159 mg/dl foi limítrofe, 160-189 $\mathrm{mg} / \mathrm{dl}$ foi alto e $\geq 190 \mathrm{mg} / \mathrm{dl}$ muito alto. O TRI foi classificado como ótimo $<150 \mathrm{mg} / \mathrm{dl}$, limítrofe $150-200 \mathrm{mg} / \mathrm{dl}$, alto $200-499 \mathrm{mg} / \mathrm{dl}$ e muito alto $\geq 500 \mathrm{mg} / \mathrm{dl}$.

Para determinar o nível glicêmico, foi utilizada a glicose (GLI), classificada conforme a American Diabetes Association ${ }^{20}$, sendo considerada desejável GLI $<100 \mathrm{mg} / \mathrm{dl}$. Os exames foram apresentados pelos usuários no momento de avaliação e reavaliação. Apenas o LDL-c foi calculado pela fórmula de Friedewald (LDL-c mg/dL $=$ colesterol total - HDL-c - Triglicerídeos $/ 5)^{21}$. A pressão arterial (PA) foi classificada conforme a 6a Diretriz Brasileira de Hipertensão ${ }^{22}$, que considera ótima PAS $<120$ e PAD $<80 \mathrm{mmHg}$, normal PAS $<130$ e PAD $<85 \mathrm{mmHg}$ e limítrofe PAS de 130-139 e PAD de 85-89 mmHg.

Para a QV, os sujeitos responderam ao WHOQOL-BREF, instrumento genérico composto por 26 questões, sendo duas gerais de QV agrupadas como DG (domínio geral) e as demais 24 divididas e organizadas em quatro domínios: DF (domínio físico), DMA (domínio de meio ambiente), DP (domínio psicológico) e DRS (domínio 
relações sociais $)^{13}$. Os resultados foram pontuados em escala de 0 a 100 , sendo que quanto maior o escore, melhor a QV. Padrão $0^{23}$ sugere uma proposta que classifica a $\mathrm{QV}$ nos seguintes cortes: qualidade de vida muito ruim (0-20); ruim (2140); nem ruim nem boa (41-60); boa (61-80); e muito boa (80-100), sendo esses pontos de corte utilizados neste estudo.

Os dados usados nas avaliações iniciais foram referentes ao período de $1^{\circ}$ de novembro de 2012 a 31 de outubro de 2013. Para as reavaliações, o período foi de $1^{\circ}$ de novembro de 2013 a 30 de outubro de 2014. Os dados foram coletados no sistema no mês subsequente ao período das reavaliações. Durante o período de adesão ao programa, os sujeitos receberam intervenções dos profissionais da equipe multiprofissional, de acordo com seu plano de cuidados individual e disponibilidade, o que não foi considerado neste trabalho, mas poderiam ser atendimentos individuais, multiprofissionais, grupos, oficinas, palestras, monitoramentos telefônicos e atividade física.

A análise estatística foi realizada no programa Statistical Package for Social Sciences (SPSS) versão 20.0. Os dados foram expostos de forma descritiva em média e desvio padrão (DV), sendo que para análise foi realizado o teste $t$ de Student para amostras paramétricas e comparação entre as médias, adotando nível de significância de 5\%.

O projeto foi aprovado pelo Comitê de Ética e Pesquisa da Universidade de Santa Cruz do Sul - UNISC.

\section{Resultados}

Os sujeitos apresentaram idade média de 59 anos $( \pm 14,2)$ na avaliação inicial, com aumento de um ano na reavaliação, sendo $77 \%$ do sexo feminino. A contratação de plano de saúde do tipo familiar é a mais frequente, $58 \%$ dos sujeitos.

Na Tabela 1 são apresentados os resultados obtidos a partir do teste t de Student. Destaca-se os TRI que, além de apresentar nível ótimo nas duas etapas, demostrou redução significativa na reavaliação $(p=0,031)$. As médias da PAS se caracterizaram como normal nas duas avaliações, enquanto a PAD foi classificada como ótima na reavaliação. Ambas demonstraram redução significativa entre as médias após a reavaliação ( $\mathrm{p}<0,001$ e $\mathrm{p}=0,013$, respectivamente). As demais variáveis não apresentaram diferença significativa, porém ressalta-se que o IMC permaneceu com a média geral classificada com obesidade grau I após a reavaliação.
Além disso, observando a classificação das variáveis por regiões, mantiveram-se os resultados, havendo diferenças apenas no IMC e na PA. O primeiro foi classificado como sobrepeso na Região 1 e obesidade grau I na Região 2, tanto na avaliação inicial como na reavaliação, e a PA foi ótima na Região 1 e normal na Região 2.

A QV foi classificada como boa, destacandose os menores escores para DG e DF. Na reavaliação, o DG foi o único que apresentou melhora significativa $(p=0,004)$. Os domínios específicos também apresentaram resultados classificados como bons, entretanto sem alterações significativas entre as avaliações, em que os domínios físicos e psicológicos apresentaram os menores escores, respectivamente (Tabela 2).

Analisando os resultados da QV por regiões, observa-se a Região 1 com melhores resultados em DG e DMA. Novamente os domínios físicos e psicológicos, respectivamente, apresentaram os menores resultados em ambas as regiões. Quanto à QV por faixa etária, mantém-se os menores escores do DG naqueles com idades $\leq 40$ anos e 41 60 anos. O DP na Região 2 aparece com destaque no grupo com menos de 40 anos, pois na avaliação inicial, quando é classificada em nem ruim nem boa $(59,9 \pm 17,6)$, apresenta o pior resultado da pesquisa, o que melhora consideravelmente na reavaliação $(68,5 \pm 14,7)$. As demais faixas trazem o DF com os menores escores, seguido pelo DP (Tabela 3). Observando por sexo, identificam-se as mulheres com resultados menores em relação aos homens em todos os domínios, equivalendo-se apenas no escore de relações sociais (dados não apresentados em tabelas).

Os sujeitos de alto risco cardiovascular apresentaram menores resultados de QV nos domínios DG e DRS, quando comparados aos sujeitos com risco baixo e risco intermediário (Tabela 4). Ao analisar os resultados por risco cardiovascular, os resultados não apresentaram diferenças estatísticas significativas, apesar de descritivamente apresentarem resultados ligeiramente melhores na maioria dos escores. Quanto à classificação do risco cardiovascular, identificou-se um número importante de sujeitos de alto risco cardiovascular na avaliação inicial (35\%) (Tabela 5), que totalizaram $37,8 \%$ na reavaliação, sendo que $60 \%$ deles se encontram na Região 2.

\section{Discussão}

No estudo houve predomínio do sexo feminino, com $61 \%$ dos integrantes na faixa etária com 
Tabela 1. Comparação de médias das variáveis de perfil na avaliação inicial (etapa 1) e na reavaliação (etapa 2).

\begin{tabular}{lrrr}
\hline Variáveis & $\begin{array}{c}\text { Etapa 1 } \bar{x} \\
(\text { DP })\end{array}$ & $\begin{array}{r}\text { Etapa 2 } \bar{x} \\
(\text { DP })\end{array}$ & p \\
\hline IMC & $30,3(5,6)$ & $30(5,3)$ & 0,340 \\
HDL-c & $56,8(13)$ & $54,8(11,7)$ & 0,375 \\
LDL-c & $116,6(30,3)$ & $113,2(31,6)$ & 0,482 \\
CT & $197,3(36)$ & $189,8(36,3)$ & 0,058 \\
TRI & $122,1(62,4)$ & $117,3(56)$ & 0,031 \\
GLI & $98,1(17,4)$ & $96(17,8)$ & 0,974 \\
PAD & $82,6(11,2)$ & $79,9(11,3)$ & $<0,001$ \\
PAS & $125,9(14)$ & $125,6(15)$ & 0,013 \\
\hline
\end{tabular}

$\bar{x}$ : média; DP: desvio padrão; significância: $\mathrm{p}<0,05$, teste $\mathrm{t}$ de Student; $\mathrm{IMC}=$ índice de massa corporal; HDL-c $=$ lipoproteína de alta densidade; LDL-c = lipoproteína de baixa densidade; $\mathrm{CT}=$ colesterol total; TRI $=$ triglicerídeos; $\mathrm{GLI}=$ glicemia $\mathrm{de}$ jejum; $\mathrm{PAD}=$ pressão arterial diastólica; $\mathrm{PAS}$ = pressão arterial sistólica.

Fonte: Dados da pesquisa.

Tabela 2. Domínios do WHOQOL-BREF e resultados relacionados à avaliação inicial e à reavaliação.

\begin{tabular}{lccc}
\hline \multirow{2}{*}{$\begin{array}{c}\text { Domínios } \\
\text { WHOQOL- }\end{array}$ BREF } & $\begin{array}{c}\text { Resultados WHOQOLação } \\
\text { inicial } \\
\bar{x}(\text { DP })\end{array}$ & $\begin{array}{c}\text { Reavaliação } \\
\bar{x}(\text { DP })\end{array}$ & $\mathbf{p}$ \\
\hline DG & $66,24(16,12)$ & $69,13(15,15)$ & 0,004 \\
DF & $66,17(13,18)$ & $65,92(15,53)$ & 0,799 \\
DMA & $71,28(9,90)$ & $70,55(13,68)$ & 0,447 \\
DP & $68,63(12,57)$ & $69,62(13,73)$ & 0,317 \\
DRS & $72,54(13,47)$ & $71,35(15,744)$ & 0,333 \\
\hline
\end{tabular}

$\overline{\bar{x}}$ : média; DP: desvio padrão; significância: $\mathrm{p}<0,05$, teste $\mathrm{t}$ de Student; DG = domínio geral; $\mathrm{DF}=$ domínio físico; DMA = domínio de meio ambiente; $\mathrm{DP}=$ domínio psicológico; $\mathrm{DRS}=$ domínio relações sociais.

Fonte: Dados da pesquisa.

mais de 61 anos, obesos e com a QV classificada como boa. No entanto, os usuários classificados como alto risco cardiovascular se autoavaliaram com os menores resultados de QV. Diante dos índices de morbimortalidade decorrentes das doenças cardiovasculares, somados ao crescente e persistente aumento da obesidade, ações de prevenção e controle dos agravos são fundamentais.

Nos domínios do WHOQOL-BREF, os resultados foram condizentes aos de outros estudos, tendo o DF apresentado os menores resultados, seguido pelo DP. Os resultados menores nesses domínios na população idosa estão associados à presença de dor e à diminuição da mobilidade, consequências do envelhecimento, e também a tratamentos relacionados a eventos de saúde, agudos ou crônicos, ou à presença de doença mental ${ }^{24-27}$.

O predomínio de mulheres também é comumente observado em outros estudos, o que pode estar associado ao maior contingente de mulheres entre os idosos, fenômeno denominado de feminização da velhice ${ }^{28}$. A maior preocupação com a saúde entre as mulheres faz com que busquem por atendimentos de saúde, tanto por uma autopercepção crítica da saúde quanto por assumirem os sintomas e realizarem mais consultas ${ }^{29}$, ao contrário dos homens, que só buscam tratamento após a manifestação dos sintomas ${ }^{19,26,30}$.

Entre os parâmetros clínicos apresentados, observou-se o IMC classificado em sobrepeso e obesidade, não demonstrando melhorias na reavaliação. Porto Alegre apresentou, no último inquérito das capitais, um percentual de 55,1\% adultos com excesso de peso ${ }^{31}$. Da mesma forma, estudos realizados em empresas, com sujeitos que receberam orientação nutricional e estímulo à prática de atividade física durante doze meses, mostraram aumento gradual e significativo do $\mathrm{IMC}^{32,33}$. Já outra pesquisa, desenvolvida em Minas Gerais, associou informação nutricional à prática regular de atividade física, concluindo que os participantes tiveram mudanças em seus hábitos alimentares e, nos que participaram regularmente, apresentaram perda de peso $^{34}$. Contudo, estudo randomizado realizado por Pekkarinen, Kaukua e Mustajoki ${ }^{35}$, na Finlândia, objetivando a redução de peso e sua manutenção na sequência, não identificou resultados diferentes entre o grupo que realizou o programa de manutenção e o controle.

Apesar de ser um grupo com predomínio de idosos e representativa parcela de sujeitos de risco cardiovascular alto e intermediário, o perfil lipídico e a glicemia apresentaram e mantiveram médias satisfatórias. O HDL-c tem importante função protetora na prevenção das doenças cardiovasculares (DCV), o que prevê níveis de HDL-c $>60 \mathrm{mg} / \mathrm{dl}$. A redução do colesterol, principalmente o LDL-c, demonstra importantes benefícios no desfecho de agravos cardiovasculares. Dessa maneira, o LDL-c apresenta metas diferenciadas conforme o risco cardiovascular: alto risco - meta LDL-c $<70 \mathrm{mg} / \mathrm{dl}$; risco intermediários - meta LDL-c $<100 \mathrm{mg} / \mathrm{dl}$; e baixo risco - meta individual ${ }^{19}$.

A PA demonstrou redução significativa entre as médias. As DCV continuam sendo a principal causa de mortalidade no Brasil, aumentando progressivamente com a elevação da PA a partir 
Tabela 3. Domínios do WHOQOL-BREF e resultados por região relacionados a avaliação inicial e reavaliação, por faixa etária.

\begin{tabular}{|c|c|c|c|c|c|c|c|}
\hline \multicolumn{2}{|c|}{ Faixa etária } & \multicolumn{3}{|c|}{ Avaliação inicial $\bar{x}$ (DP) } & \multicolumn{3}{|c|}{ Reavaliação $\bar{x}(\mathrm{DP})$} \\
\hline & & Região 1 & Região 2 & Total & Região 1 & Região 2 & Total \\
\hline \multirow{6}{*}{$\begin{array}{l}\leq 40 \\
\text { anos }\end{array}$} & $\mathrm{N}$ & 8 & 21 & 29 & 8 & 20 & 28 \\
\hline & DG & $68,8(17,7)$ & $61,9(17,9)$ & $63,8(17,8)$ & $59,4(17,4)$ & $65,6(19)$ & $63,8(18,4)$ \\
\hline & DF & $74,1(11,7)$ & $66,8(17,8)$ & $68,8(16,4)$ & $70,1(13,4)$ & $66,6(18,4)$ & $67,6(17)$ \\
\hline & DMA & $79,7(7,5)$ & $67,3(16,5)$ & $70,7(15,5)$ & $74,2(7,8)$ & $70,9(14,6)$ & $71,9(13)$ \\
\hline & DP & $77,6(12,2)$ & $59,9(17,6)$ & $64,8(17,9)$ & $63(11,9)$ & $68,5(14,7)$ & $67(13,9)$ \\
\hline & DRS & $75(14,1)$ & $73,8(20,3)$ & $74,1(18,5)$ & $74(10,4)$ & $79,2(14,4)$ & $77,7(13,4)$ \\
\hline \multirow{6}{*}{$\begin{array}{l}41 \text { a } 60 \\
\text { anos }\end{array}$} & $\mathrm{N}$ & 28 & 45 & 73 & 25 & 44 & 69 \\
\hline & DG & $62,9(19,4)$ & $62,2(18,9)$ & $62,5(19)$ & $69,6(11,6)$ & $65,2(18,9)$ & $66,8(16,7)$ \\
\hline & DF & $64,7(15,4)$ & $66,6(14,1)$ & $65,9(14,6)$ & $71,2(15,8)$ & $65,2(17,1)$ & $67,4(16,8)$ \\
\hline & DMA & $69,8(7,1)$ & $69(11,8)$ & $69,3(10,2)$ & $73,4(4,9)$ & $69,7(14)$ & $71(11,6)$ \\
\hline & DPs & $67,1(14,9)$ & $67,1(10)$ & $67,1(12)$ & $71(10,9)$ & $66,9(12,9)$ & $68,4(12,3)$ \\
\hline & DRS & $68,8(13,2)$ & $73,9(13,6)$ & $71,9(13,6)$ & $74(8,1)$ & $70,5(11,5)$ & $71,8(10,4)$ \\
\hline \multirow{6}{*}{$\begin{array}{l}\geq 61 \\
\text { anos }\end{array}$} & $\mathrm{N}$ & 75 & 74 & 149 & 78 & 76 & 154 \\
\hline & DG & $70(11,8)$ & $67,2(15,4)$ & $68,6(13,8)$ & $71,5(9)$ & $70,9(16,6)$ & $71,2(13,3)$ \\
\hline & DF & $66,4(10,2)$ & $65,2(13,1)$ & $65,8(11,7)$ & $68,8(9,4)$ & $62,7(14,9)$ & $65,8(12,7)$ \\
\hline & DMA & $73,1(5,6)$ & $71,5(9,9)$ & $72,3(8)$ & $73,1(5)$ & $71,8(8,9)$ & $72,5(7,1)$ \\
\hline & DPs & $71,4(9,1)$ & $68,9(13,1)$ & $70,2(11,3)$ & $74,5(7,4)$ & $69,7(12,2)$ & $72,2(10,2)$ \\
\hline & DRS & $72,3(7,6)$ & $72,4(15,7)$ & $72,4(12,3)$ & $73,8(5,5)$ & $72,6(12,1)$ & $73,2(9,2)$ \\
\hline
\end{tabular}

$\bar{x}$ : média; DP: desvio padrão; $\mathrm{DG}=$ domínio geral; $\mathrm{DF}$ = domínio físico; $\mathrm{DMA}=$ domínio de meio ambiente; $\mathrm{DP}$ = domínio psicológico; DRS = domínio relações sociais.

Fonte: Dados da pesquisa.

Tabela 4. Domínios do WHQOL-BREF por risco cardiovascular - avaliação inicial e reavaliação.

\begin{tabular}{lccrrrr}
\hline \multirow{2}{*}{$\begin{array}{c}\text { WHOQOL - } \\
\text { BREF (média) }\end{array}$} & \multicolumn{2}{c}{ Risco alto $\bar{x}(\mathbf{D P})$} & \multicolumn{2}{c}{ Risco intermediário $\bar{x}(\mathbf{D P})$} & \multicolumn{2}{c}{ Risco baixo $\bar{x}$ (DP) } \\
\cline { 2 - 7 } & Avaliação & Reavaliação & Avaliação & Reavaliação & Avaliação & Reavaliação \\
\hline N & 88 & 95 & 26 & 21 & 137 & 131 \\
DG & $64,6(15,5)$ & $67,5(15,9)$ & $69,7(12,3)$ & $73,2(8,2)$ & $66,7(17)$ & $70,6(14)$ \\
DF & $66(13,2)$ & $65,3(14,9)$ & $65,4(11,7)$ & $66,3(9,2)$ & $66,4(13,5)$ & $67,6(14,5)$ \\
DMA & $71,4(8)$ & $71,5(7,9)$ & $71,8(8,2)$ & $72,8(5,5)$ & $71,1(11,2)$ & $72,3(10,7)$ \\
DP & $68,8(12,6)$ & $69,8(10,8)$ & $71,6(10,1)$ & $74,4(7,4)$ & $68(12,9)$ & $70,6(12,2)$ \\
DRS & $71,2(13,2)$ & $72,4(8,7)$ & $74(9,5)$ & $72,6(9,9)$ & $72,9(14,3)$ & $74,2(10,9)$ \\
\hline
\end{tabular}

$\overline{\bar{x}}$ : média; DP: desvio padrão; $\mathrm{DG}=$ domínio geral; $\mathrm{DF}$ = domínio físico; DMA = domínio de meio ambiente; $\mathrm{DP}$ = domínio psicológico; DRS $=$ domínio relações sociais.

Fonte: Dados da pesquisa.

Tabela 5. Número de sujeitos conforme classificação do risco cardiovascular - avaliação inicial e reavaliação.

\begin{tabular}{lcc}
\hline \multicolumn{1}{c}{$\begin{array}{c}\text { Risco } \\
\text { Cardiovascular }\end{array}$} & $\begin{array}{c}\text { Avaliação } \\
\text { inicial (n) }\end{array}$ & $\begin{array}{c}\text { Reavaliação } \\
(\mathbf{n})\end{array}$ \\
\hline Alto risco & 88 & 95 \\
Risco intermediário & 26 & 26 \\
Baixo risco & 137 & 131 \\
Óbito & - & 1 \\
Sem risco calculado & - & 3 \\
Total & $\mathbf{2 5 1}$ & $\mathbf{2 5 1}$ \\
\hline Fonte: Dados da pesquisa. & &
\end{tabular}

de 115/75 mmHg. Não obstante se trata a hipertensão arterial sistêmica (HAS) como um dos principais fatores de risco (FR) modificáveis, ela tem alta prevalência e baixas taxas de controle. Nesse sentido, a orientação primária por meio de alimentação saudável, consumo controlado de sódio e álcool, ingestão de potássio, combate ao sedentarismo e ao tabagismo, associados à detecção precoce, é a forma mais efetiva de evitar a HAS e deve ser a meta prioritária dos profissionais de saúde s,8,36,37. $^{2}$. 
Os domínios (DF, DP, DMA, DRS) apresentaram resultados superiores a estudos similares realizados com usuários da saúde pública do Brasil $^{38,39}$ e classificação equivalente a um estudo empreendido com professores universitários do Rio Grande do Sul ${ }^{40}$. É importante destacar que nosso país é marcado pelas diferenças sociais, econômicas e culturais ${ }^{41}$, fatores determinantes para a $\mathrm{QV}^{42-44}$. Os sujeitos do presente estudo residem em duas regiões distintas do estado, com a Região 1 classificada em $2^{\circ}$ lugar, enquanto a Região 2 ocupa o $15^{\circ}$ lugar do Índice de Desenvolvimento Socioeconômico do Rio Grande do Sul. Contudo, os municípios das duas regiões apresentam renda per capita maior à média do estado ${ }^{18}$. Outro fator a ser destacado é que a maior parte desses usuários possuía plano de saúde do tipo familiar, o que se eleva com o aumento das faixas etárias, indicando se tratar de um grupo com condição econômica favorável. Isso explicaria o resultado de boa QV, haja vista o nível socioeconômico ser um fator determinante na $\mathrm{QV}^{42-44}$.

Não obstante os resultados do DG terem apresentado melhora significativa na reavaliação, na percepção dos sujeitos este não foi observado nos domínios específicos avaliados. Esse resultado pode ser atribuído à motivação dos sujeitos para participar do programa, como foi identificado por Baena e colaboradores ${ }^{45}$, em que as avaliações qualitativas positivas também não se refletiram em índices significativos da QV. A identificação de melhora em parâmetros de saúde sem o mesmo reflexo na avaliação da QV também foi observada por Bandini ${ }^{46}$. Esses resultados levam a reflexões sobre a abrangência do conceito de promoção da saúde, sendo necessárias ações conjuntas de diversos setores da sociedade a fim de impactar favoravelmente a QV da população e reduzir a vulnerabilidade e os riscos à saúde ${ }^{7}$.

Estudos que avaliaram a estrutura e os objetivos da promoção da saúde no setor suplementar diagnosticaram programas desenhados com expectativas para promover a melhora da QV. Entretanto, com atuação muitas vezes isolada, atentos à prevenção de doenças e ao estilo de vida saudável. Apontam, ainda, uma disponibilidade do setor para o processo de mudança e apoio às operadoras para a concepções de modelos focados em promoção da saúde, com indução a mudanças e inovações ${ }^{5,34,47}$. Dahlgren e Whitehead ${ }^{48}$ indicam que o sucesso de intervenções na saúde dos indivíduos depende da compreensão e da importância dos determinantes sociais de saúde ${ }^{49}$.

Os achados desta pesquisa sugerem a contribuição do desenvolvimento de práticas inovadoras de promoção da saúde. No entanto, este estudo apresenta algumas limitações por ter utilizado dados de prontuários eletrônicos. E por ser um estudo observacional e transversal, apresenta fragilidades, pois não proporciona resultados de um acompanhamento em longo prazo. Ainda se constitui um desafio a adesão à utilização de informações sobre QV para o planejamento e desenvolvimento de ações e políticas em saúde, o que sugere e demanda necessidade de ampliar e desenvolver novos estudos no setor suplementar.

Para tanto, é de extrema importância avaliar e melhorar os meios em que as pessoas estão inseridas, crescem e trabalham, além de considerar sua cultura. $\mathrm{Na}$ busca por ambientes saudáveis, torna-se necessário a intersetorialidade, inclusive entre o público e o privado. É urgente trazer a QV para as discussões e torná-la prioridade nas políticas públicas e privadas.

\section{Colaboradores}

HH Pohl trabalhou na concepção e na redação final. CE Beschorner contribuiu na concepção, na pesquisa e na redação. TH Lenhard trabalhou na metodologia e na estatística; AN Couto colaborou na redação final. PH Santos contribuiu na redação final. 


\section{Agradecimentos}

À Coordenação de Aperfeiçoamento de Pessoal de Nível Superior (CAPES).

\section{Referências}

1. Instituto Brasileiro de Geografia e Estatística (IBGE). Censo Demográfico 2010. Brasília: IBGE; 2010.

2. Brasil. Ministério da Saúde (MS). A vigilância, o controle e a prevenção das doenças crônicas não-transmissíveis: DCNT no contexto do Sistema Único de Saúde brasileiro. Brasília: Organização Pan-Americana da Saúde; 2005.

3. Kim CG, June KJ, Song R. Effects of a health-promotion program on cardiovascular risk factors, health behaviors, and life satisfaction in institutionalized elderly women. Int J Nurs Stud 2003; 40(4):375-381.

4. Brasil. Ministério da Saúde (MS). Secretaria de Vigilância em Saúde. Secretaria de Atenção à Saúde. Política Nacional de Promoção da Saúde. Secretaria de Atenção à Saúde. Brasília: MS; 2010.

5. Agência Nacional de Saúde Suplementar (ANS). Promoção da saúde e prevenção de riscos e doenças na saúde suplementar brasileira: resultados do laboratório de inovação. Brasília: OPAS, 2013.

6. Agência Nacional de Saúde Suplementar (ANS). Organização Pan-Americana da Saúde (OPAS). Manual técnico para promoção da saúde e prevenção de riscos e doenças na saúde suplementar. Rio de Janeiro: ANS; 2011.

7. Brasil. Ministério da Saúde (MS). Cadernos de atenção Bbsica n. 14: prevenção clínica de doença cardiovascular, cerebrovascular e renal crônica do Ministério da Saúde. Brasília: MS; 2006.

8. Brasil. Ministério da Saúde (MS). Secretaria de Vigilância em Saúde. Departamento de Análise de Situação de Saúde. Plano de ações estratégicas para o enfrentamento das doenças crônicas não transmissíveis (DCNT) no Brasil 2011-2022. Brasília: MS; 2011.

9. World Health Organization (WHO). Obesity: preventing and managing the global epidemic - report of a WHO consultation; 2004. [acesso 2017 Nov 25]. Disponível em: http://apps.who.int/iris/handle/10665/42330

10. Czeresnia D. O conceito de saúde e a diferença entre promoção e prevenção. In: Czeresnia D, Freitas CM, organizadores. Promoção da saúde: conceitos, reflexões, tendências. Rio de Janeiro: Ed. Fiocruz; 2003. p.39-53.

11. Merhy EE. A perda da dimensão cuidadora na produção da saúde. In: Campos CR, Malta DC, Reis AT, Santos AF, Merhy EE, organizadores. Sistema Único de Saúde em Belo Horizonte: reescrevendo o público. São Paulo: Xamã; 1998. p.103-20.

12. Buss PM. Promoção da saúde e qualidade de vida. Cien Saude Colet 2000; 5(1):163-177.

13. Fleck MPA, Louzada S, Xavier M, Chachamovich E, Vieira G, Santos L, Pinzon V. Aplicação da versão em português do instrumento abreviado de avaliação da qualidade de vida "WHOQOL-bref". Rev Saude Publica 2000; 34(2):178-183.

14. World Health Organization Quality of Life (WHOQOL). The development of the World Health Organization quality of life assessment instrument (the WHOQOL). In: Orley J, Kuyken W, editors. Quality of life assessment: international perspectives. Heidelberg: Springer; 1994. p. 41-60. 
15. Sestelo JAF, Souza LEPF, Bahia L. Saúde suplementar no Brasil: abordagens sobre a articulação público/privada na assistência à saúde. Cad Saude Publica 2013; 29(5):851-866.

16. Salvatori RT, Ventura CAA. A Agência Nacional de Saúde Suplementar - ANS: onze anos de regulação dos planos de saúde. Organ Soc 2012;19(62):471-487.

17. Unicef Office of Research. Child well-being in rich countries: a comparative overview. Innocenti Report Card 11. Florence: Unicef Office of Research; 2013.

18. FEE Dados. Corede Vale do Rio Pardo e Corede Vale do Taquari. Rio Grande do Sul: Fundação de Economia e Estatística, 2013. [acesso 2018 Out 20]. Disponível em: http://www.fee.rs.gov.br/perfilsocioeconomico/coredes/detalhe/?corede $=$ Vale + do + Rio + Pardo

19. Sociedade Brasileira de Cardiologia (SBC). V Diretrizes da Sociedade Brasileira de Cardiologia. V Diretrizes Brasileiras sobre Dislipidemias e Diretriz de Prevenção da Aterosclerose do Departamento de Aterosclerose da Sociedade Brasileira de Cardiologia. Arq Bras Cardiol 2013; 101(4 - Supl. 1): p. 1-22.

20. American Diabetes Association (ADA). Standards of Medical Care in Diabetes - 2014. Diabetes Care 2014; 37(Suppl. 1):s14-s80.

21. Friedewald W, Levi R, Fredrickson D. Estimation of the concentration of low-density lipoproteins cholesterol in plasma without use of the ultracentrifuge. Clin Chem 1972; 18:499-502.

22. Sociedade Brasileira de Cardiologia (SBC). VI Diretrizes Brasileiras de Hipertensão. Arq Bras Cardiol 2010; 95(1 - Supl. 1):1-51.

23. Padrão MB. Avaliação da qualidade de vida de doadores vivos após o transplante renal utilizando os instrumentos SF-36 e WHOQOL-bref [dissertação]. São Paulo: Faculdade de Ciências Médicas; 2008.

24. Ramaprasad D, Rao NS, Kalyanasundaram S. Disability and quality of life among elderly persons with mental illness. Asian J Psychiatr 2015; 18:31-36.

25. Bruijn MAAM, Synhaeve NE, Rijsbergen MWA, Leeuw FE, Mark RE, Jansen BPW, Kort PLM. Quality of life after young ischemic stroke of mild severity is mainly influenced by psychological factors. J Stroke Cerebrovasc Dis 2015; 24(10):2183-2188.

26. Estrella-Castillo DF, Gomez-de-Regil L. Quality of life in Mexican patients with primary neurological or musculoskeletal disabilities. Disabil Health J 2015; 9(1):127-133.

27. Castellano JM, Penalvo JL, Bansilal S, Fuster V. Promotion of cardiovascular health at three stages of life: never too soon, never too late. Rev Esp Cardiol 2014; 67(9):731-737.

28. Almeida AV, Mafra SCT, Silva EP, Kanso S. A feminização da velhice: em foco as características socioeconômicas, pessoais e familiares das idosas e o risco social. Textos \& Contextos 2015; 14(1):115-131.

29. Verbrugge LM. The twain meet: empirical explanations of sex differences in health and mortality. $J \mathrm{He}$ alth Soc Behav 1989; 30:282-304.

30. Pinheiro RS, Viacava F, Travassos CEB, Santos A. Gênero, morbidade, acesso e utilização de serviços de saúde no Brasil. Cien Saude Colet 2002; 7(4):687-707.
31. Brasil. Ministério da Saúde (MS). Vigitel Brasil 2017: vigilância de fatores de risco e proteção para doenças crônicas por inquérito telefônico - estimativas sobre frequência e distribuição sociodemográfica de fatores de risco e proteção para doenças crônicas nas capitais dos 26 estados brasileiros e no Distrito Federal em 2017. Brasília: MS; 2018.

32. Bandini M. Impacto de ações de promoção da saúde incluídas no Programa de Controle Médico de Saúde Ocupacional de empresa do ramo alimentício [tese]. São Paulo, Universidade de São Paulo; 2006.

33. Ciorlia LA, Godoy MF. Fatores de risco cardiovascular e mortalidade. Seguimento em longo prazo (até 20 anos) em programa preventivo realizado pela medicina ocupacional. Arq Bras Cardiol 2005; 85(1):20-25.

34. Rodrigues AT, Silva KL, Sena RR. Health promotion programs within supplementary healthcare in Belo Horizonte, MG, Brazil: concepts and practices. Interface (Botucatu) 2015; 19(54):455-66.

35. Pekkarinen T, Kaukua J, Mustajoki P. Long-term weight maintenance after a 17 -week weight loss intervention with or without a one-year maintenance program: a randomized controlled trial. J Obes 2015; 651460.

36. Sociedade Brasileira de Cardiologia (SBC). I Diretriz Brasileira de Prevenção Cardiovascular. Arq Bras Cardiol 2013; 101(6 - Supl. 2):1-63.

37. European Society of Cardiology. European guidelines on cardiovascular disease prevention in clinical practice. Eur Heart J 2003; 24(17):1601-1610.

38. Vitorino LM, Paskulin LMG, Vianna LAC. Quality of life among older adults resident in long-stay care facilities. Rev Latino-Am Enfermagem 2012; 20(6):11861195.

39. Miranzi SSC, Ferreira FS, Iwamoto HH, Pereira GA, Miranzi MAS. Qualidade de vida de indivíduos com diabetes mellitus e hipertensão acompanhados por uma equipe de saúde da família. Texto contexto - enferm 2008; 7(4):672-679.

40. Koetz L, Rempel C, Perico E. Qualidade de vida de professores de instituições de ensino superior comunitárias do Rio Grande do Sul. Cienc Saude Colet 2013; 18(4):1019-1028.

41. Azevedo ALS, Silva RA, Tomasi E, Quevedo LA. Doenças crônicas e qualidade de vida na atenção primária à saúde. Cad Saude Publica 2013; 29(9):1774-1782.

42. Cheng H, Green A, Wolpert M, Deighton J, Furnham A. Factors influencing adult quality of life: findings from a nationally representative sample in the UK. Personality and Individual Differences 2014; 68:241246.

43. Barrera G, Cases T, Bunout D, Maza MP, Leiva L. Rodriguez JM, Hirsch S. Associations between socioeconomic status, aging and functionality among older women. Geriatric Nursing. 2014 Oct: 1-5.

44. Méjean C, Droomers M, van der Schouw YT, Sluijs I, Czernichow S, Grobbee DE, Bueno-de-Mesquita HB, Beulens JW. The contribution of diet and lifestyle to socioeconomic inequalities in cardiovascular morbidity and mortality. International Journal of Cardiology. 2013; 168:5190-5195. 
45. Baena CP, Muccillo-Baisch AL, Almeida TL, Rocha C, Franco OS, Olmedo D, Soares MCF. Impacto de um programa piloto de promoção da saúde para trabalhadores marítimos de rebocadores. Rev bras saude осир 2011; 36(124):288-296.

46. Bandini M. Impacto de ações de promoção da saúde incluídas no Programa de Controle Médico de Saúde Ocupacional de empresa do ramo alimentício (tese). São Paulo: Universidade de São Paulo; 2006.

47. Barbosa ML, Celino SDM, Costa GMC. The Family Health Strategy in the supplementary sector: adoption of the primary care model in a self-management company. Interface (Botucatu) 2015; 19(55):11011108.

48. Dahlgren G, Whitehead M. Policies and strategies to promote social equity in health: background document to WHO - strategy paper for Europe. Stockholm: Institute for Futures Studies; 1991.

49. Buss PM, Pellegrini FA. A saúde e seus determinantes sociais. Physis 2007; 17(1):77-93.

Artigo apresentado em 21/10/2018

Aprovado em 18/11/2019

Versão final apresentada em 20/11/2019

Editores-chefes: Romeu Gomes, Antônio Augusto Moura da Silva 